\title{
Exploring Earth's Matter Effect in High-Precision Long-Baseline Experiments
}

\author{
Masoom Singh ${ }^{a, b, *}$ and Sanjib Kumar Agarwalla ${ }^{b, c, d}$ \\ ${ }^{a}$ Utkal University, Vani Vihar, Bhubaneswar, Odisha 751004, India, \\ ${ }^{b}$ Institute of Physics, Sachivalaya Marg, Sainik School Post, Bhubaneswar 751005, India \\ ${ }^{c}$ Homi Bhabha National Institute, Training School Complex, Mumbai 400094, India \\ ${ }^{d}$ International Centre for Theoretical Physics, Strada Costiera 11, Trieste 34151, Italy \\ E-mail: masoom@iopb.res.in (ORCID: 0000-0002-8363-7693), \\ sanjib@iopb.res.in (ORCID: 0000-0002-9714-8866)
}

The Earth's matter effect is going to play a crucial role in measuring the unknown three-flavor neutrino oscillation parameters at high confidence level in future high-precision long-baseline experiments. We observe that owing to the new degeneracies among the most uncertain oscillation parameters $\left(\delta_{\mathrm{CP}}, \theta_{23}\right)$ and the average Earth's matter density $\left(\rho_{\mathrm{avg}}\right)$ for the $1300 \mathrm{~km}$ baseline, the sensitivity of the upcoming Deep Underground Neutrino Experiment (DUNE) to establish Earth's matter effect reaches only about $2 \sigma$ C.L. for all possible choices of oscillation parameters. We notice that the current uncertainty in $\delta_{\mathrm{CP}}$ degrades the measurement of $\rho_{\text {avg }}$ more as compared to $\theta_{23}$. To lift these degeneracies, we explore the possible complementarity between DUNE and Tokai to Hyper-Kamiokande (T2HK/JD) facility with a second detector in Korea, popularly known as T2HKK or JD+KD setup. While DUNE uses a wide-band beam with an on-axis detector, T2HKK setup plans to use a narrow-band beam with two off-axis detectors: one in Japan and other in Korea. We exhibit how the high-precision measurement of $\delta_{\mathrm{CP}}$ in JD+KD setup and the information on $\rho_{\text {avg }}$ coming from DUNE can reduce the impact of these degeneracies in both $\left(\rho_{\text {avg }}-\delta_{\mathrm{CP}}\right)$ and $\left(\rho_{\text {avg }}-\theta_{23}\right)$ planes. We show that the combined data from DUNE and JD+KD setups can establish Earth's matter effect at more than $6 \sigma$ C.L. irrespective of both the choices of mass hierarchy: normal $(\mathrm{NH})$ and inverted $(\mathrm{IH}), \delta_{\mathrm{CP}}$, and $\theta_{23}$. With the help of this combined data set, we can measure the average matter density $\left(\rho_{\text {avg }}\right)$ with a relative $1 \sigma$ precision of around $11.2 \%(9.4 \%)$ assuming true $\mathrm{NH}(\mathrm{IH})$ and $\delta_{\mathrm{CP}}=-90^{\circ} / 90^{\circ}$.

\footnotetext{
*** The European Physical Society Conference on High Energy Physics (EPS-HEP2021), ***

*** 26-30 July $2021 * * *$

*** Online conference, jointly organized by Universität Hamburg and the research center DESY ***
}

\footnotetext{
${ }^{*}$ Speaker
} 


\section{Complementarity between DUNE and T2HKK (JD+KD) Setups}

In this work, we explore the interesting complementarity between the two next generation high-precision long-baseline experiments DUNE and T2HKK (JD+KD) in establishing the Earth's matter effect [1] by rejecting the vacuum oscillation. The DUNE far detector (a $40 \mathrm{kt}$ LArTPC) will receive an on-axis, high-intensity, wide-band neutrino beam covering both first and second oscillation maxima with a baseline of $1300 \mathrm{~km} \mathrm{[2].} \mathrm{On} \mathrm{the} \mathrm{other} \mathrm{hand,} \mathrm{the} \mathrm{T2HKK} \mathrm{setup} \mathrm{plans}$ to house its first far detector (187 kt, water Cherenkov detector) in Japan (JD) at a distance of 295 $\mathrm{km}$ from J-PARC and to deploy an another $187 \mathrm{kt}$, water Cherenkov detector in Korea (JD+KD) at a baseline of $1100 \mathrm{~km}$ [3]. The Japanese (Korean) detector will observe an off-axis $\left(2.5^{\circ}\right)$, narrow-band beam covering the first (second) oscillation maximum. We expect a high-precision measurement of $\delta_{\mathrm{CP}}$ and a conclusive evidence for leptonic CP violation from this JD+KD setup, which has much less matter effect. On the other hand, DUNE feels a substantial matter effect due to its larger baseline and energies as compared to the JD+KD setup. Therefore, the combined data from DUNE and JD+KD may establish the Earth's matter effect at high C.L. by reducing the impact of possible degeneracies among the oscillation parameters $\left(\delta_{\mathrm{CP}}, \theta_{23}\right)$ and the average Earth's matter density $\left(\rho_{\text {avg }}\right)$. In this work, we discuss several interesting issues along this direction.

\section{Establishing Earth's matter effect}

We perform our simulations using the GLoBES software [4]. We generate the prospective data with the following choices of oscillation parameters: $\sin ^{2} \theta_{23}=[0.44,0.5,0.56], \sin ^{2} 2 \theta_{13}=$ $0.085, \sin ^{2} \theta_{12}=0.307, \delta_{\mathrm{CP}}$ in the range $-180^{\circ}$ to $180^{\circ}, \Delta m_{31}^{2}=2.5(-2.4) \times 10^{-3} \mathrm{eV}^{2}$ for NH (IH), $\Delta m_{21}^{2}=7.4 \times 10^{-5} \mathrm{eV}^{2}$, and the average matter density $\left(\rho_{\text {avg }}\right)=2.86 \mathrm{~g} / \mathrm{cm}^{3}$ for all the three $(\mathrm{JD}, \mathrm{KD}$, and DUNE) baselines. The statistical significance of the long-baseline experiments to establish the
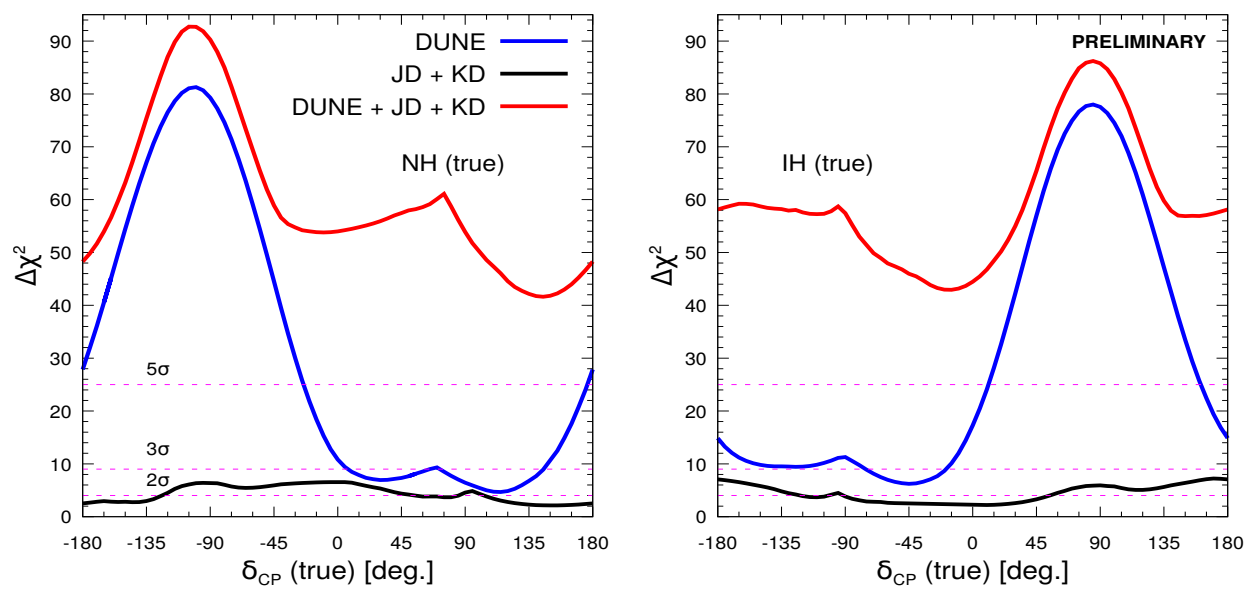

Figure 1: The sensitivity of JD+KD (black curves), DUNE (blue curves), and the combined DUNE+JD+KD setup (red curves) in establishing the Earth's matter effect as a function of true $\delta_{\mathrm{CP}}$ assuming true $\mathrm{NH}(\mathrm{IH})$ in the left (right) panel. We consider $\sin ^{2} \theta_{23}$ (true) $=0.5$ and marginalize over $\sin ^{2} \theta_{23}=[0.4: 0.6], \delta_{\mathrm{CP}}=\left[-180^{\circ}: 180^{\circ}\right]$, and $\Delta m_{31}^{2}= \pm[2.36: 2.64] \times 10^{-3}$ in the fit.

Earth's matter effect by refuting the vacuum oscillation is defined as follows

$$
\Delta \chi^{2}=\min _{\left(\vec{\gamma}, \lambda_{1}, \lambda_{2}\right)}\left\{\chi^{2}\left(\rho_{\mathrm{avg}}^{\text {true }} \neq 0\right)-\chi^{2}\left(\rho_{\mathrm{avg}}^{\text {test }}=0\right)\right\},
$$


where $\vec{\gamma}=\left\{\theta_{23}, \delta_{\mathrm{CP}}, \Delta m_{31}^{2}\right\}$ is the set of oscillation parameters on which marginalization is performed and $\lambda_{1}, \lambda_{2}$ are the systematic pulls [5] on signal and background, respectively. In Fig.1 we observe that DUNE (blue lines) itself can establish the matter effect for about $45 \%$ choices of true $\delta_{\mathrm{CP}}$ at $5 \sigma$ C.L. for both true $\mathrm{NH}$ and IH. On the other hand, the JD+KD setup (black lines) alone has much less sensitivity towards the Earth's matter effect. When we combine the performance of DUNE and JD+KD, we observe a significant enhancement in the sensitivity and Earth's matter effect can be established with more than $6 \sigma$ C.L. (red lines) for all possible choices of true $\delta_{\mathrm{CP}}$ and for both $\mathrm{NH}$ and $\mathrm{IH}$. We see this improvement in the sensitivity for the unfavorable choices of true $\delta_{\mathrm{CP}}$ (around $0^{\circ}$ to $180^{\circ}$ for true $\mathrm{NH}$ and $-180^{\circ}$ to $0^{\circ}$ for true $\mathrm{IH}$ ) because the data from JD+KD setup reduces the impact of marginalization over test $\delta_{\mathrm{CP}}$ while analyzing the data from DUNE.

\section{Precision measurement of $\rho_{\text {avg }}$}

The statistical significance to measure $\rho_{\text {avg }}$ in a given experiment is defined as

$$
\Delta \chi_{\mathrm{PM}}^{2}\left(\rho_{\mathrm{avg}}\right)=\chi^{2}\left(\rho_{\mathrm{avg}}\right)-\chi_{0}^{2},
$$

where we obtain $\chi^{2}\left(\rho_{\text {avg }}\right)$ by performing a fit to the prospective data with $\rho_{\text {avg }}=2.86 \mathrm{~g} / \mathrm{cm}^{3}$ and $\chi_{0}^{2}$ is the minimum value of $\chi^{2}\left(\rho_{\text {avg }}\right)$ considering $\rho_{\text {avg }}$ in the range of 1.5 to $4 \mathrm{~g} / \mathrm{cm}^{3}$. Fig. 2 shows

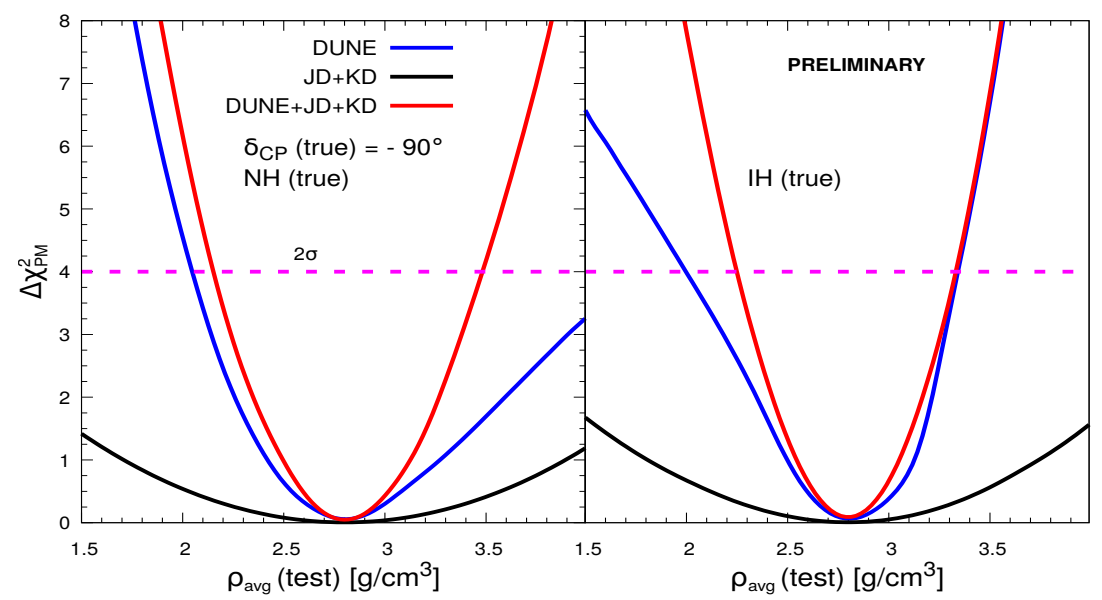

Figure 2: Left (Right) panel shows the achievable precision in the measurement of $\rho_{\text {avg }}$ for JD + KD (black lines), DUNE (blue lines), and DUNE+JD+KD (red lines) assuming true $\mathrm{NH}(\mathrm{IH})$. Here, we consider true $\delta_{\mathrm{CP}}=-90^{\circ}$ and $\sin ^{2} \theta_{23}=0.5$. In the fit, we marginalize over $\sin ^{2} \theta_{23}, \delta_{\mathrm{CP}}$, and $\Delta m_{31}^{2}$ (see Fig. 1 caption).

that the JD+KD setup alone offers a relative $1 \sigma$ precision in $\rho_{\text {avg }}$ of around $40 \%(35 \%)$ for true $\mathrm{NH}(\mathrm{IH})$ assuming $\delta_{\mathrm{CP}}($ true $)=-90^{\circ}$ and $\sin ^{2} \theta_{23}$ (true) $=0.5$. The same quantity for DUNE setup alone is around $15 \%(12 \%)$. Interestingly, when we combine the data from these two high-precision experiments, the achievable precision in $\rho_{\text {avg }}$ reaches to $11.2 \%(9.4 \%)$.

\section{Degeneracies in test $\left(\rho_{\mathrm{avg}}-\delta_{\mathrm{CP}}\right)$ and test $\left(\rho_{\mathrm{avg}}-\theta_{23}\right)$ Planes}

The black curves in left (right) panel of Fig. 3 shows that the JD+KD setup alone can measure $\delta_{\mathrm{CP}}\left(\theta_{23}\right)$ quite precisely while having almost no sensitivity towards $\rho_{\text {avg }}$ due to their shorter

baselines. Whereas the DUNE setup alone can constrain the allowed ranges in $\rho_{\text {avg }}$ and can provide reasonable measurements of $\delta_{\mathrm{CP}}$ and $\theta_{23}$ (blue curves). When we combine the data from 

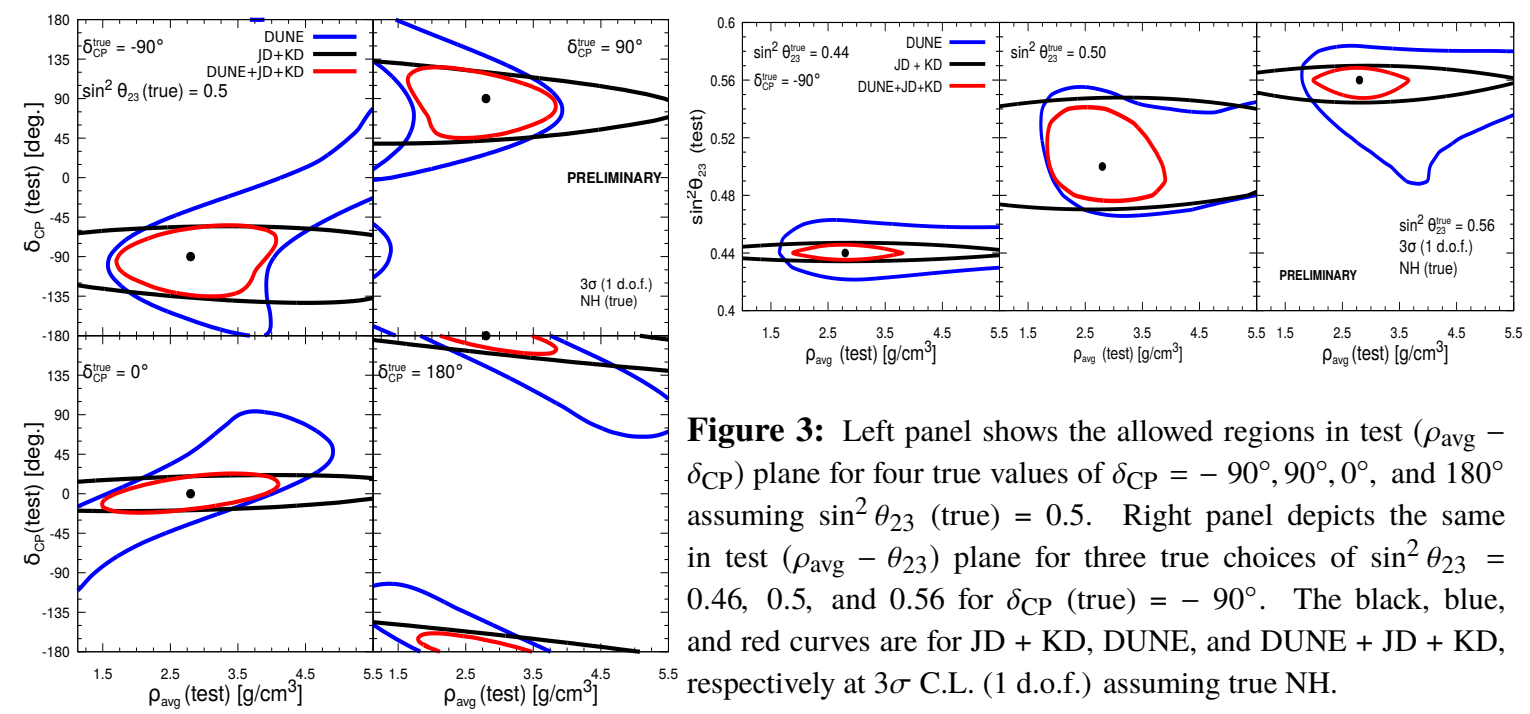

Figure 3: Left panel shows the allowed regions in test $\left(\rho_{\text {avg }}-\right.$ $\left.\delta_{\mathrm{CP}}\right)$ plane for four true values of $\delta_{\mathrm{CP}}=-90^{\circ}, 90^{\circ}, 0^{\circ}$, and $180^{\circ}$ assuming $\sin ^{2} \theta_{23}$ (true) $=0.5$. Right panel depicts the same in test $\left(\rho_{\text {avg }}-\theta_{23}\right)$ plane for three true choices of $\sin ^{2} \theta_{23}=$ $0.46,0.5$, and 0.56 for $\delta_{\mathrm{CP}}$ (true) $=-90^{\circ}$. The black, blue, and red curves are for JD + KD, DUNE, and DUNE + JD + KD,

respectively at $3 \sigma$ C.L. (1 d.o.f.) assuming true $\mathrm{NH}$.

these two setups, we see a considerable reduction in the allowed ranges in both $\left(\rho_{\text {avg }}-\delta_{\mathrm{CP}}\right)$ and $\left(\rho_{\text {avg }}-\theta_{23}\right)$ planes (red curves) due to the complementary information from these two experiments.

\section{Conclusion}

DUNE with $1300 \mathrm{~km}$ baseline has significant matter effect and can measure $\delta_{\mathrm{CP}}$ and $\theta_{23}$ with reasonable precision exploiting the information on oscillation pattern at several $L / E$ values. On the other hand, with a relatively shorter baseline and high statistics JD offers an unmatched sensitivity to the $\delta_{\mathrm{CP}}$ free from matter effect. KD with a roughly four times baseline than JD has some sensitivity to Earth's matter effect and provides crucial information on $\delta_{\mathrm{CP}}$ around the second oscillation maxima. In this work, for the first time, we show how the complementary features between DUNE and JD+KD setups can play an important role to establish the Earth's matter effect at more than $6 \sigma$ C.L. for any values of oscillation parameters. The complementary informations coming from DUNE and JD+KD setups also play an important role to provide a high-precision measurement of $\rho_{\text {avg }}$ and to reduce the allowed regions in $\left(\rho_{\text {avg }}-\delta_{\mathrm{CP}}\right)$ and $\left(\rho_{\text {avg }}-\theta_{23}\right)$ planes considerably.

Acknowledgements : M.S. acknowledges financial support from DST, Govt. of India (DST/INSPIRE Fellowship/2018/IF180059). S.K.A. acknowledges financial support from DAE, DST, DST-SERB, Govt. of India, and INSA. The numerical simulations are performed using SAMKHYA: High-Performance Computing Facility at Institute of Physics, Bhubaneswar.

\section{References}

[1] G.L.Fogli et al. Phys. Rev. D 66(5) Sep 2002, [hep-ph/0206162].

[2] T. Alion et al. Experiment Simulation Configurations used in DUNE CDR 6.2016.

[3] Abe et al. Progress of Theoretical and Experimental Physics, 2018(6), Jun 2018.

[4] P. Huber et al. Computer Physics Communication 177(5) 432-438, Sep 2007.

[5] S. P. Mikheyev and A. Y. Smirnov, Resonant amplification of $v$ oscillations in matter and solar neutrino spectroscopy Il Nuovo Cimento C 9(1):17-26, January, 1986. 